\title{
Corneal Epithelial Cell Movement in Humans
}

\author{
M. A. LEMP and W. D. MATHERS \\ Washington, DC
}

\begin{abstract}
Summary
Evidence is presented which supports the centripetal movement of epithelial cells in the normal corneal epithelium. This movement is not, however, uniform and is influenced by various factors including corneal topography, surface disease states and lid shearing forces. We have studied epithelial morphology with corneal specular microscopy and have demonstrated altered morphology in keratoconjunctivitis sicca, neurotrophic keratitis, and contact lens wearing. Following penetrating keratoplasty, we found a vortex keratopathy in 70 per cent of patients up to two years after surgery. We also found pallisading of epithelial cells around sutures which indicated centripetal movement of epithelial cells around islands of stability created by obstructions. The eyelid also alters epithelial migration and turnover by increasing exfoliation from shearing forces. We advance a new hypothesis that the driving force in the central epithelial cell movement is the preferential loss of surface cells by exfoliation from the central apex secondary to the shearing forces of the upper lid.
\end{abstract}

The subject of epithelial cell movement in the cornea has been of interest for many years. In the late nineteenth century, Peters ${ }^{1}$ described the rapid sliding of epithelial cells after corneal injury; subsequent studies have demonstrated a sliding of adjacent epithelial cells with a pseudopodal extension of their processes into the defect shortly after injury. ${ }^{2}$ It has also been noted that there is a 'lag period' of approximately one day in which there is a cessation of DNA synthesis and basal cell mitoses in an area surrounding the wound. ${ }^{3}$ Approximately 24 hours after injury, DNA synthesis begins in the underlying stromal cells and a wave of epithelial cell mitosis moves towards the wound crater finally involving the basal cells which cover the wound. Eventually this activity results in a new epithelium of normal thickness. Epithelial sliding is dependent upon the presence of intracellular glycogen and can be retarded by glycogen depletion with certain metabolic poisons. ${ }^{3}$
Epithelial healing, therefore, in response to injury, consists of at least two stages; an initial sliding process which covers the defect rapidly and then a second phase of mitotic activity resulting in a thickening of the epithelium. It has been further observed in the healing of epithelial defects that re-epithelialisation proceeds first downward, subsequently horizontally and only finally from below. ${ }^{5}$

Dua and Forrester ${ }^{6}$ followed the pattern of closure of epithelial abrasions in humans employing fluorescein stain and planimetry. They noted that all abrasions involving the central cornea and in which the limbus was intact healed in a similar manner. There was a non-uniform advancement of two, three or more foci of epithelial sheets producing multiple convex fronts which ultimately met giving rise to ' $y$ ' or double ' $y$ ' shaped patterns from which epithelial cells form a whorl pattern.

In other studies of the repair of epithelial 
defects of the cornea, it has been noted that the conjunctiva can play a significant role as a source for epithelial cell renewal in the cornea. Thoft, et al ${ }^{7.89}$ have detailed the movement of epithelial cells to recover corneal epithelial defects. Recently, Danjo, et al. ${ }^{10}$ studied the mitotic rate and goblet cell density of conjunctival epithelium following total or central corneal epithelial removal one day following injury (total removal of corneal and limbal epithelium). The mitotic rate of the surrounding perilimbal conjunctiva was found to be ten times the normal. When central five or ten millimeter epithelial abrasions were created, an increase in the mitotic rate of the perilimbal epithelium was noted to be three to four times normal. Goblet cell density in this area also decreased. These studies present strong evidence that conjunctival epithelium can play a major role in the resurfacing of large and even limited cornal epithelial injuries. These studies also point out that there can be a substantial movement of cells from the periphery to the centre of the cornea. Recent studies employing electronmicroscopy present morphological evidence that such conjunctivally-derived 'corneal epithelium' can remain morphologically conjunctiva for many months.'

These studies of corneal injury however, create a set of circumstances different from that which occurs in the normal renewal of corneal epithelium. It has been thought for many years that the basal cells of the corneal epithelium account for the major regenerative activity of the corneal epithelium under normal circumstances. Employing tritiated thymidine and autoradiography in the rabbit model, Hanna and O'Brien ${ }^{12}$ found that one in seventy-five central basal epithelial cells was in a pre-mitotic synthetic state over a two hour period. From this they calculated the average life cycle for a new epithelial cell to range between three and a half to seven days in the rabbit. They further reckoned, however, that a mitotic rate based on the above data was insufficient to account for the replenishment of epithelial cells lost from the surface. In 1971, Davanger and Evensen, ${ }^{13}$ two ophthalmic researchers working in Uganda, focused attention on the limbus as a probable source of major epithelial regenera- tion. They noted that this area is characterised by deep involutions of epithelium which are surrounded by richly vascularised papillae (the palisades of Vogt). This area covered by the basal cells is large due to increased interfacial area and these basal cells are adjacent to a well-developed capillary net. These authors have suggested that the limbus is the major source of epithelial cell regeneration and that epithelial cells produced in the limbus are carried centripetally towards the central cornea. Furthermore, melanocytes are not normally present in the cornea but exist in abundance in the neighbourhood of blood vessels at the limbus, particularly in heavily pigmented individuals. ${ }^{15}$ (Recent evidence supporting the concept of centripetal movement of epithelial cells lies in the observation that there is a radial arrangement of hemidesmosomal alignment along the basement membrane in normal murine eyes).${ }^{14}$ It is a common clinical observation that in heavily pigmented patients there are often radially distributed pigment lines extending from the corneal limbus, variable distances along the surface of the cornea suggesting that this pigment is pulled along as epithelial cells move towards the centre of the cornea.

Based on these observations and other experimental data, Thoft and Friend proposed the ' $x, y, z$ hypothesis' which states that corneal epithelial cell maintenance is dependent upon a continuous, centripetal migration of epithelial cells as well as proliferation and subsequent exfoliation from the corneal surface. ${ }^{16}$ In a recent experiment, Buck ${ }^{17}$ marked the peripheral cornea with a rotating needle containing a mixture of India ink and thorium dioxide. After seven days, the marker was visible in the underlying corneal stroma; the marker contained in epithelium had moved away from the stromal marks some distance centrally. This migration was seen in the superficial and wing cells of the corneal epithelium but was not present in basal cells. The notion of greater regenerative power in the peripheral cornea was given further credence by the recent observation of Ebato, et al. ${ }^{18}$ who found that explants of peripheral cornea showed greater outgrowth and a higher mitotic rate than those from the central cornea.

In the last decade, it has been found that all 
epithelial cells contain keratin. There are over twenty different types of keratin within epithelial cells. ${ }^{19}$ The introduction of monoclonally-produced keratin specific antibodies has provided a powerful investigative tool in the study of epithelial cell behaviour. Investigators have used this technology to study the 'keratin profiles' of different types of epithelial cells. Attention has been focused on 'stem cells'. ${ }^{20}$ These are believed to represent a stable population of primitive cells undergoing regular cell division, in turn giving rise to a population of intermediately differentiated cells called transient amplifying (TA) cells. These TA cells are thought to divide subsequently, several times, amplifying the number of stem cell derived cells before subsequent terminal differentiation into tissue specific epithelial cells, e.g. corneal epithelial cells. Recent data employing anti-keratin antibodies have demonstrated that corneal basal cells are more differentiated in their keratin expression than are limbal basal cells. ${ }^{21}$ The schema of 'stem cells' giving rise to TA cells which then subsequently give rise to fully differentiated cells is established as a working hypothesis buttressed by a substantial body of experimental data.

In an extensive, descriptive clinical study in

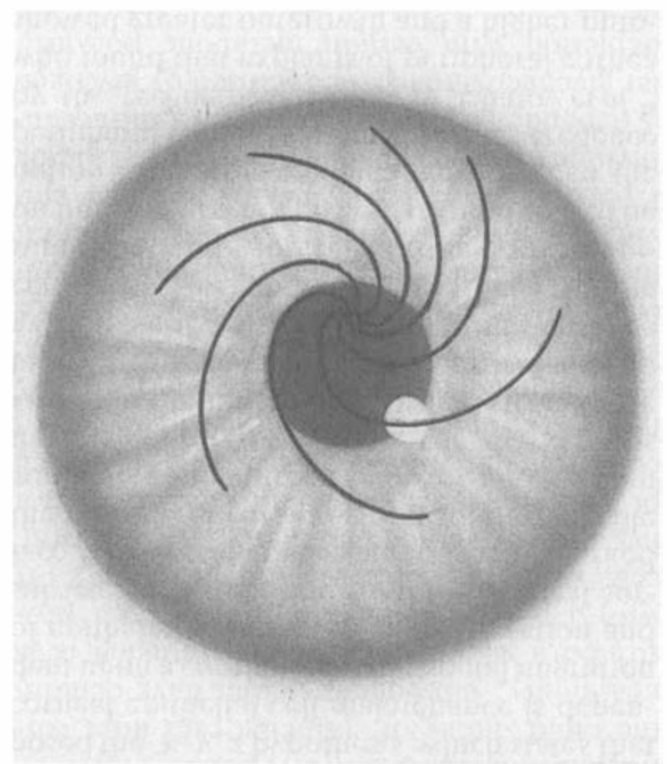

Fig. 1. Diagram of the vortex pattern in the human corneal epithelium.
1973, Bron ${ }^{22}$ noted a variety of clinical conditions in which swirled, radiating lines (vortex patterns) in the corneal epithelium were seen to be a common feature (Fig. 1). Conditions listed included Fabry's disease, toxic keratopathies, such as those seen with chloroquine and amiodarone, striate melanocytosis, healing corneal abrasions and conditions associated with iron lines in the cornea, band keratopathy, corneal oedema and the periphery of penetrating keratoplasties. Bron advanced the hypothesis that the resemblance in patterns in these diverse clinical conditions was dependent upon the growth and movement properties of the epithelium rather than any specific stimulus. He postulated a centripetal 'pigment slide' observed in corneas of highly pigmented individuals. Such pigment would, therefore, form a 'marker' for epithelial cell movement. In lightly pigmented individuals, alternative markers might be accumulated intracellular metabolic products such as those seen in Fabry's disease, chloroquine or amiodarone keratopathy. The inferiorly decentred apical vortex pattern is consistent with a predominantly downward cellular movement. The constant feature noted is a radial, centripetal slide from the limbus to the corneal centre.

From the preceding, it is clear that there is accumulating evidence that epithelial cell regeneration is largely centred in the peripheral cornea and limbus, that there is a general, centripetal movement from the periphery to the centre of the cornea and that this pattern is operative in the normal renewal of epithelial cells in the cornea. This general notion implies a continuous uniform process across the corneal surface. The uniformity of cell movement in the cornea is, however, called into question by a number of clinical situations in which there appear to be areas of corneal epithelium which remain stable over long periods of time. Such areas can be detected by noting visible intracellular markers such as iron, melanin or accumulated metabolic products. Iron lines of the cornea have been noted for many years; both Hudson ${ }^{23}$ and Stahli ${ }^{24}$ described a brown line, inferior to the corneal centre, appearing in normal subjects. Gass ${ }^{25}$ has noted that this curvilinear line extends along the line of lid closure in more than three-quarters of 
patients over the age of 50 . This line is formed by the intraepithelial accumulation of iron. Similar iron lines have been noted at the base of keratoconus, ${ }^{24}$ in advance of the head of a pterygium,${ }^{27}$ adjacent to filtering blebs ${ }^{28}$ in a trough central to sutures in a penetrating keratoplasty, ${ }^{29}$ in encircling corneal scars,$^{30}$ sur- rounding a Salzman's nodule, ${ }^{31}$ and in irregularly scarred corneas ${ }^{30}$ (Fig. 2).

More recently, similar iron lines have appeared around the edge of lenticules in epikeratoplasty, in keratophakia and hyperopic keratomileusis $^{32}$ and in a stellate form between incision scars in radial keratotomy. ${ }^{33}$

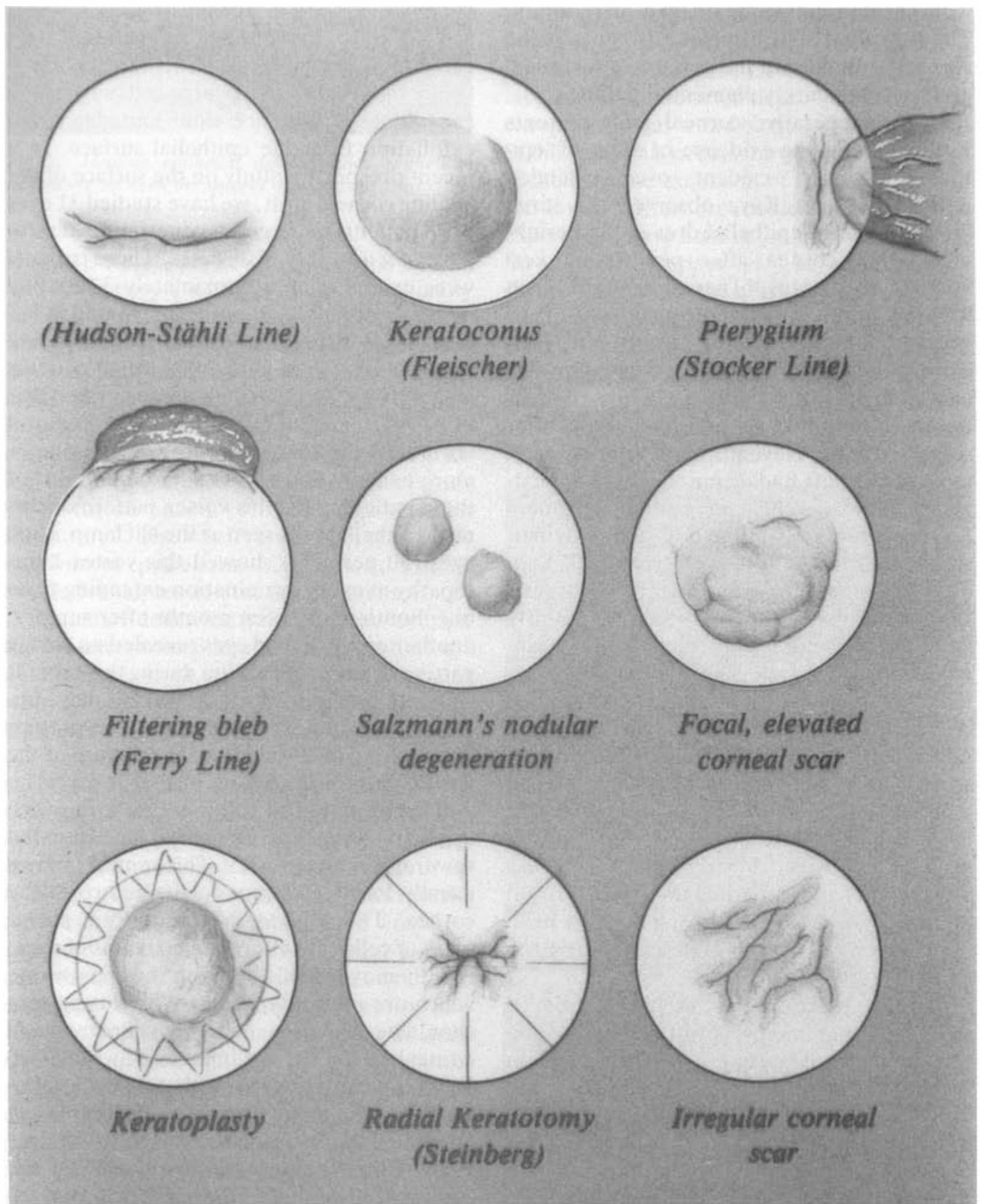

Fig. 2. Patterns of iron lines seen with various clinical entities. 
A feature common to all these areas (excepting the Hudson-Stahli line) is their location in areas of depression on the corneal surface.

A line similar in appearance to the HudsonStahli line but originating at the limbus and radiating towards the central cornea has been observed in many darkly pigmented patients with chronic inflammation and after injury. This so-called striate melanosis can also be seen extending onto the surface of a corneal graft in some darkly pigmented patients.

The postoperative corneal graft presents further interesting evidence of areas of 'epithelial stability' evident over extended periods of time. Kaye observed the serial development of epithelial dots in the peripheral donor cornea after penetrating keratoplasty in humans. ${ }^{34}$ These dots were seen in $47 \%$ of grafts studied prospectively. They occurred in a peripheral area of the graft corresponding to an annular depression just central to the sutures. The mean time of onset for these dots was six and half weeks after surgery. By the eleventh week after surgery, most of the dots had acquired a grey appearance and by week 20, a fine epithelial pigment line was observed. These dots generally persisted until the sutures were removed. Subsequent to suture removal, a centripetal movement of the dots was seen over a thirty day period before they completely disappeared. Histopathologic examination of epithelium removed from these areas revealed degenerated cells lying at the level of the wing and superficial epithelial cells. Recently, fifteen cases of a distinctive 'hurricane' shaped epitheliopathy occurring postoperatively in corneal grafts, have been reported. ${ }^{35}$ These changes were seen as early as several weeks after surgery and were thought to be the result of a medication toxicity, giving rise to a healing vortex pattern such as described by Bron earlier. ${ }^{22}$

Over the past six years, we have employed colour specular microscopy of the corneal epithelium to study surface cell morphology in the human cornea. ${ }^{36,37}$ This technique while difficult to perform, can provide significant morphological information in a relatively non-invasive manner. The technique of performing this test is described elsewhere. We have studied the normal, central corneal sur- face, that of patients with keratoconjunctivitis sicca, ${ }^{38}$ neurotrophic keratitis, the corneal surface of patients with extended-wear contact lenses $^{39}$ and, more recently, the surface of the healing corneal graft. ${ }^{40}$ We have demonstrated that there is a statistically significant shift in epithelial size in patients with keratoconjunctivitis sicca, demonstrating smaller, central cells. In contrast, in patients with extended-wear contact lenses, there is a statistically significant shift to large cells suggesting a prolonged residence time and decreased exfoliation from the epithelial surface. In a recent prospective study on the surface of the healing corneal graft, we have studied 31 eyes of 28 patients undergoing penetrating keratoplasty for a variety of diseases. These patients were examined at approximately 3 monthly intervals, for fifteen months, beginning at 1-3 months after surgery. At least three separate examinations were performed on all patients in this group. These studies demonstrated that in 22 of 31 grafts $(71 \%)$ there was a central vortex pattern observed with CSM in one or more examinations. In less than one-third of these patients, was this vortex pattern of corneal epithelial cells seen at the slit lamp. Nine eyes (30 per cent) showed this vortex keratopathy at every examination extending from one month to eighteen months after surgery; another group of nine eyes revealed no vortex pattern at any examination during this time. It was interesting to note, however, that subsequent to suture removal, the vortex pattern disappeared in all subjects. The picture of the vortex pattern is striking in that it reveals a pattern of epithelial cell movement in which epithelial cells are elongated and pointed towards a central vortex. This central point is usually located inferior to the centre of the cornea. This suggests a non-uniform movement of cells with alternating streams of more rapidly moving cells between those pathways which are relatively stable. Why this pattern should be evident in such a high percentage of corneal grafts is not immediately obvious. What is clear, however, is that just central to the sutures in many corneal grafts, there is an annular depression. This, in the same area where Kaye's dots are frequently seen. If one studies epithelium around sutures, postgrafting, one sees a radial arrangement of cells 


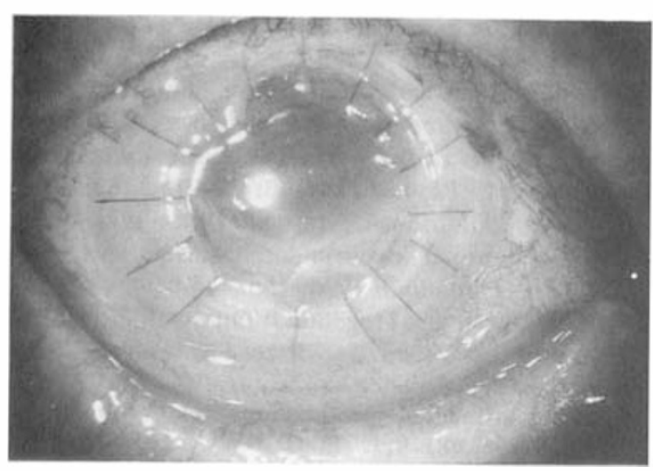

Fig. 3. Healing epithelial defect shows epithelium advancing between the sutures of a corneal graft.

around sutures, suggesting a cellular movement in between sutures but not immediately surrounding the sutures. This in effect creates a series of 'gates' for epithelial movement from the periphery to the centre, separated by areas of relative epithelial stability. Further supporting evidence for the 'gate' concept in corneal graft is seen in Figure 3 in which healing of an epithelial defect in a corneal graft shows the advancing epithelial edge between the sutures. The one feature common to all elements of epithelial stability noted are areas of relative depression on the corneal surface.

Recent evidence has pointed to the fact that the lid exerts considerable force on the corneal surface, both with normal blinking and with forced blinking. Doane, ${ }^{41}$ employing high speed cinematography has demonstrated a $1-6 \mathrm{~mm}$ retromovement of the globe with each blink. More recently, Frueh ${ }^{42}$ placed a pressure-sensitive device within a contact lens and measured the force of the upper lid on the cornea. A forced blink registered values of up to $200 \mathrm{~g}$. As the upper lid moves downward, the area of maximum applied force would be expected to be towards the central cornea near the corneal apex. Lesser forces would be expected toward the limbus, inferiorly and superiorly. It is known that the lower lid does not move upward significantly during a blink. A differential gradient in the lid pressure would then be expected (Fig. 4). Such a gradient in shearing forces from the lid could set up differences in surface cell exfoliation. Such exfoliation would be expected to be greatest at the corneal centre.
In situations with abnormal corneal topography, e.g. the healing corneal graft, with areas of epithelial depression in situations in which an iron line has been noted in corneal depressions, one would expect the lid forces to be lesser in these depressed areas than in the surrounding areas. It seems likely, therefore, that this could account for the nonuniform movement of cells becasue of a decrease in exfoliation from the corneal surface in these areas of depression. The one exception to this woud be that of the HudsonStahli line. This occurs, however, at the point of the extreme excursion of the upper lid and the lower lid, an area which would also be expected to have little in the way of a shearing force from the upper lid. The effect of such shearing forces on the normal corneal surface would be expected to be exaggerated in conditions in which the lubricity between the lid and corneal surface is decreased, such as keratoconjunctivitis sicca. Indeed, our CSM studies have demonstrated a statistically significant

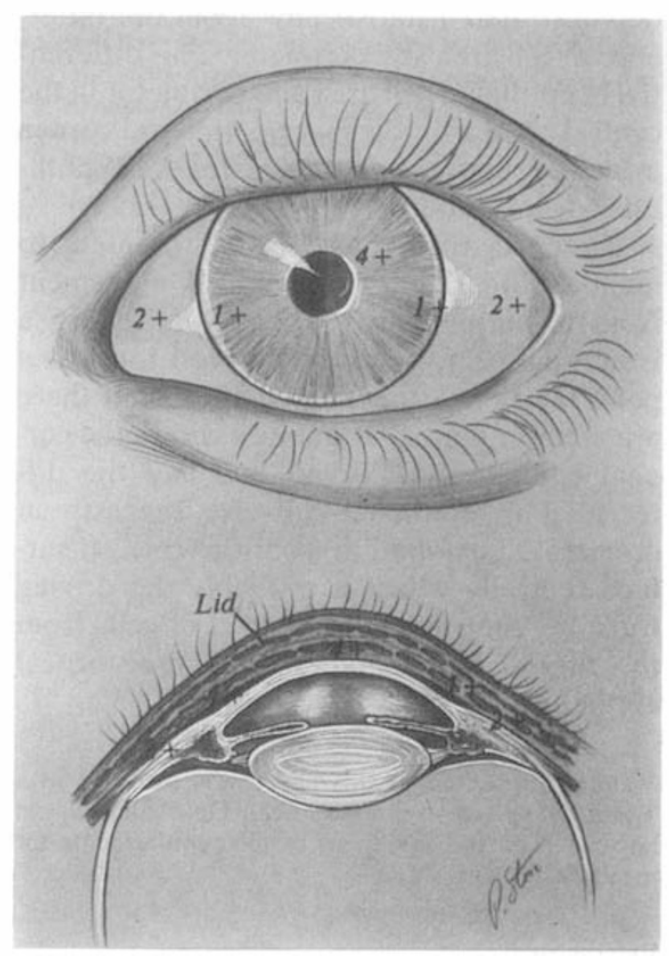

Fig. 4. Pressure differential generated by the eyelid over the corneal surface graded from plus one to plus four. 
decrease in cell size, suggesting newly emerged cells predominate the central cornea in patients with KCS. ${ }^{38}$ This further suggests an increased exfoliative rate from the surface of the cornea in KCS. We advance a new hypothesis in which epithelial cell movement on the cornea results from a differential sliding of epithelium from the periphery to the centre; that the driving force in this central epithelial cell movement in the preferential loss of surface cells by exfoliation from the corneal apex secondary to the shearing forces of the upper lid.

It is generally thought that newly emerged corneal surface cells are smaller than cells that are resident for longer periods of time on the corneal surface. ${ }^{43}$ If our hypothesis is correct, one would expect to see a difference in epithelial cell size from the central cornea to more peripheral locations. Preliminary data employing CSM in normal subjects in which specular photomicrographs were taken in the central cornea, the superior peripheral cornea and the inferior peripheral cornea have demonstrated a statistically significant difference in cell area and perimeter. This differential in epithelial cell area and perimeter in the central cornea $v s$. the more peripheral cornea is further supporting evidence for the hypothesis advanced here.

Evidence then has been presented to demonstrate that epithelial cell movement from the periphery to the centre of the cornea is non-uniform, seems to proceed towards a point inferior to the corneal apex, that there are areas of epithelial stability within the corneal epithelial cell population and the differential in epithelial cell size suggests an accelerated exfoliation from the corneal surface centrally which is probably the driving force for centripetal movement of cells from the limbus in the renewal of the corneal epithelium.

Material presented in this paper was included in a Thesis presented to the American Ophthalmological Society in partial fulfilment of the requirements for membership.

\footnotetext{
References

'Peters A: Regeneration des Epithels der Cornea. Inaugural Dissertation, 1885, Bonn.

${ }^{2}$ Buschke W: Morphologic changes in cells of corneal
}

epithelium in wound healing. Arch Ophthalmol 1949, 41: 306-16.

${ }^{3}$ Friedenwald JS and Buschke W: The influence of some experimental variables on the epithelial movements in the healing of corneal wounds. $J$ Cell Comp Physiol 1944, 23: 95-107.

${ }^{4}$ Kuwabara T, Perkins DG, Cogan DG: Sliding of the epithelium in experimental corneal wounds. Invest Ophthalmol 1976, 15: 4-14.

5 Madgyaszaz A and Hegedus J: On the epithelialization of the cornea. Szemeszet 1967, 104: 66-9.

${ }^{6}$ Dua HS and Forrester JV: Clinical patterns of corneal epithelial wound healing. Am J Ophthalmol 1987, 104: 481-9.

7 Thoft RA and Friend J: Biochemical transformation of regenerating ocular surface epithelium. Invest Ophthalmol 1977, 16: 14-20.

${ }^{8}$ Kinoshita S, Friend J, Thoft RA: Sex chromatin of donor corneal epithelium in rabbits. Invest Ophthalmol Vis Sci 1981, 21: 434-41.

${ }^{9}$ Friend J, Kinoshita S, Thoft RA: Biphasic cell proliferation in transdifferentiation of conjunctival to corneal epithelium in rabbits. Invest Ophthalmol Vis Sci 1983, 24: 1008.

${ }^{10}$ Danjo S, Friend J, Thoft RA: Conjunctival epithelium in healing of corneal epithelial wounds. Invest Ophthalmol Vis Sci 1987, 28: 1445-9.

${ }^{11}$ Buck RC: Ultrastructure of conjunctival epithelium replacing corneal epithelium. Current Eye Res 1986, 5: 149-59.

12 Hanna C and O'Brien JE: Cell production and migration in the epithelial layer of the cornea. Arch Ophthalmol 1960, 64: 536-9.

${ }^{13}$ Davanger $\mathrm{M}$ and Evensen A: Role of the pericorneal papillary structure in renewal of corneal epithelium. Nature 1971, 229(286): 560-1.

${ }^{14}$ Buck RC: Hemidesmosomes of normal and regenerating mouse corneal epithelium. Virchows Arch B Cell Pathology 1982, 41: 1-16.

${ }_{15}$ Henkind P: Pigment Cell Migration. MSc Thesis, New York University, 1964.

${ }^{16}$ Thoft RA and Friend J: The X, Y, Z hypothesis of corneal epithelial maintenance. Letter to the editor. Invest Ophthalmol Vis Sci 1983, 24: 1442-3.

${ }^{17}$ Buck RC: Measurement of centripetal migration of normal corneal epithelial cells in the mouse. Invest Ophthalmol Vis Sci 1985, 26: 1296-9.

${ }^{18}$ Ebato B, Friend J, Thoft RA: Comparison of central and peripheral human corneal epithelium in tissue culture. Invest Ophthalmol Vis Sci 1987, 28: 1450-6.

${ }^{19}$ Sun T-T, Eichner R, Nelson WG, Tseng SC, Weiss RA, Jarvinen M. Woodcock-Mitchell J: Keratin classes: molecular markers for different types of epithelial differentiation. J Invest Dermatol 1983, 81: $109 \mathrm{~s}-15 \mathrm{~s}$.

20) Lavker RM and Sun T-T: Epidermal stem cells. $J$ Invest Dermatol 1983, 81-121s-7s.

${ }^{21}$ Schermer A, Galvin S, Sun T-T: Differentiationrelated expression of a major $64 \mathrm{~K}$ corneal keratin in vivo and in culture suggests limbal location of corneal epithelial stem cells. J Cell Biol 1986, 103: 49-62. 
22 Bron AJ: Vortex patterns of the corneal epithelium. Trans Ophthalmol Soc UK 1973, 93: 455-72.

${ }^{23}$ Hudson AC: A note on certain peculiar pigmentary markings in the cornea. $R$ London Ophthalmol Hosp Rep 1911, 18: 198-9.

${ }^{24}$ Stahli J: Uber den Fleischerschen Ring beim Keratoconus und eine typische Epithelpigmentation der normalen Kornea. Klin Monatsblatt Augenheilk 1918, 60: 721-8.

${ }^{25}$ Gass JDM: The iron lines of the superficial cornea: Hudson-Stahli Line, Stocker's Line and Fleischer's ring. Arch Ophthalmol 1964, 71: 34858.

${ }^{26}$ Fleischer B: Uber Keratoconus und eigenartiger Figurenbildreng en der Cornea. Munchen Med Wschr 1906, 53: 625-9.

${ }^{27}$ Stocker FW and Prindle RE: A new type of pigment line in the cornea. Am J Ophthalmol 1944, 27: 341-5.

${ }^{28}$ Ferry AP: A 'new' pigment line of the superficial cornea. Occurrence in patients with filtering blebs. Arch Ophthalmol 1968, 79: 142--5.

${ }^{29}$ Mannis MJ: Iron deposition in the corneal graft: Another corneal iron line. Arch Ophthalmol 1983, 101: 1858-61.

${ }^{30}$ Barraquer-Somers E, Chan C-C, Green WR: Corneal epithelial deposition of iron. Ophthalmology 1983, 90: 729-30.

${ }^{31}$ Reinach NW and Baum J: A corneal pigmented line associated with Salzmann's nodular degeneration. Am J Ophthalmol 1981, 91: 677-8.

32 Koenig SB, MacDonald MB, Yamaguchi T, Friedlander M, Ishii Y: Corneal iron lines after refractive keratoplasty. Arch Ophthalmol 1983, 101: 1862-5.

${ }^{33}$ Steinberg EB, Wilson LA, Waring GO, Lynn MJ,
Coles WH: Stellate iron lines in the corneal epithelium after radial keratotomy. Am J Ophthalmol 1984, 98: 416-21.

${ }^{34}$ Kaye DB: Epithelial response in penetrating keratoplasty. Am J Ophthalmol 1980, 89: 381-7.

${ }^{35}$ Mackman GS, Polack FM, Sydrys L: Hurricane keratitis in penetrating keratoplasty. Cornea 1983, 2: 31-4.

${ }^{36}$ Lemp MA, Guimaraes RQ, Mahmood MA, Wong $\mathrm{S}$, Blackman $\mathrm{HJ}$ : In vivo surface morphology of the human cornea by color microscopy. Cornea 1983, 2: 295-7.

37 Wong S, Rodriguez MA, Blackman HJ, Guimaraes R, Lemp MA: Color specular microscopy of disorders invoving the corneal epithelium. Ophthalmology 1984, 91: 1176-83.

${ }^{38}$ Lemp MA, Gold JB, Wong S, Mahmood MA, Guimaraes R: An in vivo study of corneal surface morphologic features in patients with keratoconjunctivitis sicca. Am J Ophthamol 1984, 98: 426-8.

${ }^{39}$ Lemp MA and Gold JB: The effects of extended wear hydrophilic contact lenses on the human corneal epithelium. Am J Ophthalmol 1986, 101: 274-7.

40) Lemp MA: The surface of the corneal graft: in vivo color specular microscopic study in the human. Trans Am Ophthalmol Soc, (submitted).

${ }^{41}$ Doane M: Interaction of eyelids and tears in corneal wetting and the dynamics of the normal human eyeblink. Am J Ophthalmol 1980, 89: 507-16.

${ }^{42}$ Frueh BR: Graves' eye disease: Orbital compliance and other physical measurements. Trans Am Ophthalmol Soc 1984; 82: 492-598.

${ }^{43}$ Pfister RR: The normal surface of corneal epithelium: a scanning electron microscopic study. Invest Ophthalmol 1973, 12: 654-68. 